\title{
BMJ Open Is sarcopenia a risk factor for reduced diaphragm function following hepatic resection? A study protocol for a prospective observational study
}

\author{
Gregory van der Kroft (D) ," Sebastian Johannes Johannes Fritsch (D) , \\ S S Rensen, ${ }^{3,4}$ Steffen Wigger, ${ }^{1}$ Christian Stoppe, ${ }^{5}$ Andreas Lambertz, ${ }^{1}$ \\ Ulf Peter Neumann, ${ }^{1,3}$ S W M Olde Damink, ${ }^{1,3,4}$ Christian S Bruells ${ }^{5}$
}

To cite: van der Kroft G, Fritsch SJJ, Rensen SS, et al. Is sarcopenia a risk factor for reduced diaphragm function following hepatic resection? A study protocol for a prospective observational study. BMJ Open 2021;11:e053148. doi:10.1136/ bmjopen-2021-053148

- Prepublication history and additional supplemental material for this paper are available online. To view these files, please visit the journal online (http://dx.doi.org/10.1136/ bmjopen-2021-053148).

SWMOD and CSB contributed equally.

Received 11 May 2021 Accepted 25 August 2021

Check for updates

(c) Author(s) (or their employer(s)) 2021. Re-use permitted under CC BY-NC. No commercial re-use. See rights and permissions. Published by BMJ.

For numbered affiliations see end of article.

Correspondence to Dr Gregory van der Kroft; gregory.kroft@gmail.com

\section{ABSTRACT}

Introduction Sarcopenia is associated with reduced pulmonary function in healthy adults, as well as with increased risk of pneumonia following abdominal surgery. Consequentially, postoperative pneumonia prolongs hospital admission, and increases in-hospital mortality following a range of surgical interventions. Little is known about the function of the diaphragm in the context of sarcopenia and wasting disorders or how its function is influenced by abdominal surgery. Liver surgery induces reactive pleural effusion in most patients, compromising postoperative pulmonary function. We hypothesise that both major hepatic resection and sarcopenia have a measurable impact on diaphragm function. Furthermore, we hypothesise that sarcopenia is associated with reduced preoperative diaphragm function, and that patients with reduced preoperative diaphragm function show a greater decline and reduced recovery of diaphragm function following major hepatic resection. The primary goal of this study is to evaluate whether sarcopenic patients have a reduced diaphragm function prior to major liver resection compared with non-sarcopenic patients, and to evaluate whether sarcopenic patients show a greater reduction in respiratory muscle function following major liver resection when compared with non-sarcopenic patients.

Methods and analysis Transcostal B-mode, M-mode ultrasound and speckle tracking imaging will be used to assess diaphragm function perioperatively in 33 sarcopenic and 33 non-sarcopenic patients undergoing right-sided hemihepatectomy starting 1 day prior to surgery and up to 30 days after surgery. In addition, rectus abdominis and quadriceps femoris muscles thickness will be measured using ultrasound to measure sarcopenia, and pulmonary function will be measured using a handheld bedside spirometer. Muscle mass will be determined preoperatively using CT-muscle volumetry of abdominal muscle and adipose tissue at the third lumbar vertebra level (L3). Muscle function will be assessed using handgrip strength and physical condition will be measured with a short physical performance battery . A rectus abdominis muscle biopsy will be taken intraoperatively to measure proteolytic and mitochondrial activity as well as inflammation and redox status. Systemic inflammation and sarcopenia biomarkers will be assessed in serum acquired perioperatively.
Strengths and limitations of this study

- This study prospectively investigates the impact of sarcopenia on diaphragm function of patients undergoing right-sided hemihepatectomy in a homogenic surgical cohort.

- Patient physical condition and pulmonary function is extensively and objectively recorded.

- This study uses novel non-invasive speckle ultrasound to measure diaphragmatic strain as a measure of diaphragm effort.

Ethics and dissemination This trial is open for recruitment. The protocol was approved by the official Independent Medical Ethical Committee at Uniklinik (Rheinish Westphälische Technische Hochschule (RWTH) Aachen (reference EK309-18) in July 2019. Results will be published via international peer-reviewed journals and the findings of the study will be communicated using a comprehensive dissemination strategy aimed at healthcare professionals and patients.

Trial registration number ClinicalTrials. gov (EK309-18); Pre-results.

\section{INTRODUCTION}

Sarcopenia and muscle wasting are known risk factors for outcome following hepatic resection. ${ }^{1-5}$ Sarcopenia has also been shown to be associated with reduced pulmonary function and increased risk of pneumonia following abdominal surgery. ${ }^{6-10}$ Postoperative pneumonia has been shown to prolong hospital admission and increase in-hospital mortality following a range of surgical interventions, including major abdominal and upper gastrointestinal surgery. ${ }^{11-15}$ In addition, patients undergoing partial hepatectomy frequently develop reactive pleural effusion and postoperative pneumonia incidences above 10\%. ${ }^{11}{ }^{13-18}$ Besides medical implications, pulmonary complications 
constitute a significant burden to healthcare systems by increasing healthcare costs. ${ }^{13} 16$

Cachexia and sarcopenia are interrelated wasting disorders, the pathogenesis of which is complex due to its multifactorial nature and is characterised by a negative protein and energy balance that is driven by a variable combination of reduced food intake and abnormal metabolism. ${ }^{19}$ The known associations between sarcopenia and pulmonary outcome are based on appendicular muscle mass measurements, or measurements at the third lumbar vertebra (L3). Interestingly, very little is known about the function of the diaphragm in the context of sarcopenia and wasting disorders or how its function is influenced by abdominal surgery. ${ }^{21}{ }^{21}$ Some preclinical animal studies have shown that sarcopenia is associated with atrophy of diaphragmatic muscle fibres, and that ageing is related to a decline in diaphragmatic function. ${ }^{22-24}$ Clinical studies in the context of prolonged mechanical ventilation of acutely ill patients have focused on diaphragm function using ultrasound technology and have shown that prolonged ventilation can lead to diaphragm atrophy which is associated with worse clinical outcome. ${ }^{25-27}$

Transcostal B-mode and M-mode ultrasound is a noninvasive technique which has been used to measure diaphragm function. ${ }^{27-31}$ Diaphragm inspirational amplitude (DIA) has been shown to decrease significantly following open cholecystectomy. ${ }^{28}$ Fractional thickening (FT) of the diaphragm has been used in previous studies to quantify effort of the diaphragm. ${ }^{31}{ }^{32}$ Recently, Deniz et $a l^{21}$ demonstrated that sarcopenic elderly patients have significantly reduced diaphragm thickness and pulmonary function than non-sarcopenic elderly. Twodimensional deformation ultrasound or speckle tracking (ST) has emerged as a tool which can be used to evaluate diaphragm function in a way that is highly correlated to the gold standard trans diaphragmatic pressure measurements. ${ }^{33}$ ST finds its origins in myocardial function ultrasound and enables distinct assessment of cardiac muscle function. ${ }^{34}$ The grey value pattern in ultrasound images remains relatively constant for any small region in muscle tissue, this is called a speckle. In the ST technique, a defined cluster of speckles is tracked from one frame to another during a contractile cycle. This enables the twodimensional quantification of diaphragm deformation (strain).

We hypothesise that both major hepatic resection and sarcopenia have a measurable impact on diaphragm function. Furthermore, we hypothesise that sarcopenia is associated with reduced preoperative diaphragm function, and that patients with reduced preoperative diaphragm function show a greater decline and reduced recovery of diaphragm function following right-sided hemihepatectomy.

The primary goal of this study is to evaluate whether sarcopenic patients have a reduced diaphragm function prior to major liver resection compared with nonsarcopenic patients, and to evaluate whether sarcopenic patients show a greater reduction in respiratory muscle function following right-sided hemihepatectomy when compared with non-sarcopenic patients. The secondary objective of this study is to investigate proteolytic activation and markers of mitochondrial activity in muscle tissue, as well as systemic inflammation markers of sarcopenic and non-sarcopenic patients.

\section{METHODS AND ANALYSIS}

\section{Inclusion and exclusion criteria}

This study will entail a prospective observational singlecentre study, analysing consecutive patients undergoing open right-sided hemihepatectomy with biliary reconstruction between the ages of 18 years and 80 years. Surgery is performed as part of standard care. Only patients undergoing open right-sidedhemi hepatectomy with biliary reconstruction will be included in the study to achieve a homogeneous study cohort. Exclusion will be on the basis of American Anesthesiology Association classification IV or higher, liver cirrhosis child grade B or higher, end-stage renal disease requiring dialysis, severe heart disease New York Heart Association class IV, preexisting pulmonary conditions including, chronic obstructive pulmonary disease, asthma, history of pulmonary surgery, history of pulmonary embolism, smoking, pleural effusion occupying more than $1 / 3$ of the pleural space, neurological disorders leading to paraparesis of the upper or lower limbs or known muscular dystrophic disorders. Patients will consecutively be assigned to a sarcopenic and non-sarcopenic group based on CT-muscle quantification and hand grip strength (definitions will be detailed below). Sarcopenia stratification will be performed by an investigator blinded for the diaphragm ultrasound and pulmonary function testing and vice versa.

Patients will receive postoperative analgesia in the form of standard postoperative analgesia protocols. Administration of analgesia and pain scoring (Visual Analogue Scales) will be recorded by the primary investigator to correct for pain-associated restriction of pulmonary function.

\section{Blinding of results}

To ensure unbiased ultrasound evaluation and pulmonary function testing, the investigator performing diaphragm ultrasound and pulmonary function testing will be blinded for the sarcopenia stratification. Thus, investigator 1 will perform stratification of patients into sarcopenic and non-sarcopenic groups based on L3 CT and handgrip strength measurements. Investigator 2 will perform ultrasound and pulmonary function testing as well as physical condition testing and blood sampling.

\section{ENDPOINTS}

\section{Primary endpoints}

Differences in diaphragm kinetics as measured by ST ultrasound (eg, deformation\% (strain), deformation 
velocity (strain rate), and dimensional measures which are FT and range of DIA) and abdominal muscle kinetics (eg, deformation\%, deformation velocity, FT and DIA, see above) between sarcopenic and non-sarcopenic study groups prior to elective right-sided hemihepatectomy.

\section{Secondary endpoints}

Longitudinal changes from baseline values in diaphragm and abdominal muscle (rectus abdominis muscle) kinetics (eg, deformation\%, deformation velocity, FT and DIA), and the occurrence and quantification of pleural effusion in the postoperative phase across sarcopenic and nonsarcopenic groups following elective right-sided hemihepatectomy as measured by ST ultrasound compared with each other and preoperative values.

\section{Tertiary endpoints}

Perioperative quantitative and qualitative evaluation of a panel of biomarkers associated with inflammation, mitochondrial function and the pathogenesis of sarcopenia (see online supplemental appendix 1) in the sarcopenic and non-sarcopenic groups.

\section{DEFINING SARCOPENIA AND PHYSICAL CONDITION}

Sarcopenia will be defined as either low estimated muscle mass measured by CT-muscle volumetry or reduced muscle function measured by handgrip strength, or reduced physical condition as defined by the European Working Group on Sarcopenia in Older People (EWGSOP). ${ }^{35}$

\section{CT-muscle quantification}

CT-muscle volumetry of abdominal muscle and adipose tissue at L3 will be performed on abdominal CT scans. This method provides an accurate estimation of wholebody muscle mass as well as the detection of sarcopenia. ${ }^{36}$ These scans will be taken for routine diagnostic purposes and will not lead to additional radiation exposure of patients for the purpose of this study. Muscle volumetry at the L3 level will be corrected for patient stature. Using gender and body mass index specific cut offs as described by Martin et al (L3-skeletal muscle index of $<55 \mathrm{~cm}^{2} / \mathrm{m}^{2}$ for men or $<39 \mathrm{~cm}^{2} / \mathrm{m}^{2}$ for women), patients will be categorised as sarcopenic or non-sarcopenic. ${ }^{36}{ }^{37}$ In addition, other body composition parameters, such as muscle radiation attenuation, visceral fat mass and subcutaneous fat mass will be evaluated for association with our primary outcome variable.

\section{Functional muscle measurement (handgrip strength)}

In addition to muscle mass, a measurement of muscle function will be performed. A handgrip strength test will be performed to ascertain muscle strength on both hands with the elbow flexed at $90^{\circ}$. Three repeats will be performed and the highest value will be used for analysis. The value of the dominant hand will be used for stratification into sarcopenic and non-sarcopenic groups. This method has been validated for the detection of sarcopenic patients and cut-off points for detection have been defined by the EWGSOP. ${ }^{35}$

\section{Physical condition test}

To assess physical performance, a short physical performance battery (SPPB) test will be performed. The SPPB has been recently recommended by an international working group for use as a functional outcome measure in clinical trials in frail older persons and is recommended as part of the detection algorithm for sarcopenia by the EWGSOP ${ }^{38}$ Furthermore, cut-off points have been defined by the EWGSOP.

\section{ULTRASOUND}

Patients will undergo a transcostal ultrasound in the supine position 1 day prior to surgery, as well as on the first, third, fifth and seventh postoperative day. The ultrasound transducer will be positioned longitudinally to the anterior axillary line between the 9th and 11th intercostal space. In this location, the diaphragm is identified as a threelayered structure just superficial to the liver, consisting of a relatively non-echogenic muscular layer bounded by the echogenic membranes of the diaphragmatic pleura and peritoneum. A $10 \mathrm{~s}$ recording will be made at maximum frame rate for analysis. In addition, pleural effusion will be observed, and if present, quantified.

B-mode and M-mode Ultrasound FT will be measured using a $13 \mathrm{MHz}$ linear array transducer. Diaphragmatic thickness will be measured at end-expiration (Tdi,ee) and peak inspiration (Tdi,pi; that is, peak thickness value during inspiration) as the distance between the diaphragmatic pleura and the peritoneum using M-mode. Measurements of Tdi,ee and Tdi,pi will always be made on two respiratory cycles visualised in a single M-mode. Diaphragm thickening during inspiration (DTdi) will be taken as the difference between Tdi,pi and Tdi,ee. Diaphragm thickening fraction will be defined as the percentage change in diaphragm thickness during inspiration (computed from the quotient of DTdi and Tdi,ee). ${ }^{31}{ }^{32}$ DIA will be determined using M-mode ultrasound. ${ }^{27}$

\section{ST analysis}

Following the ultrasound recording of diaphragm kinetics, images will be analysed using software to ascertain strain rate, FT and range of motion.

Strain describes the relative change in length between an initial reference state (L0) and compressed/shortened state $(\mathrm{L})$. The conventional strain is defined as: $\varepsilon$ $=(\mathrm{L}-\mathrm{L} 0) / \mathrm{L} 0$. Positive strain means stretching, whereas negative strain means shortening. To investigate strain, a region of interest will be placed between the echogenic line of the peritoneum and pleural line. Strain rate indicates the rate of deformation as follows: $\varepsilon^{\prime}=\mathrm{d} \varepsilon / \mathrm{dt}$. Strain rate is an instantaneous measurement not requiring a relation to a reference state. The strain will be measured 
as longitundinal strain using commercially available software (EchoPac, GE Healthcare).

\section{Lung function testing}

Following ultrasound, diaphragm kinetics will be recorded during normal breathing on both sides, and once at pi-max (peak inspiratory pressure). Pi-max will be measured with a portable hand-held spirometer (Micro Respiratory Pressure Meter (RPM) handheld spirometer) which patients must breath through at maximum capacity. This will provide broad insight into the general respiratory capacity, as well as at maximum effort.

\section{STUDY TIME PLAN}

Patients will be asked to participate in the study at the time of first contact in the outpatient clinic, in the weeks prior to surgery. Data collection will commence 1 day prior to surgery and will end 6 months after discharge from the hospital. Data will be collected at eight time points (T1 though T8) during this period, namely 1 day prior to surgery, during surgery, and at day 1, 3, 5 and 7 postoperatively. Thirty days after surgery, data collection will be performed during a routine postoperative check-up. Six-months after surgery, a telephone questionnaire focused on physical activity and quality of life (36Item Short Form Health Survey Questionnaire, SF-36) will be performed (see study flowchart figure 1). Dropouts from the study as well as loss to follow-up and missing data will be recorded and stated in the final draft of the manuscript.

\section{- T1 (1 day preoperative)}

First data collection. Patients will be stratified as being sarcopenic or non-sarcopenic. An additional blood sample will be taken and frozen ( $5 \mathrm{~mL}$ EDTA) from the antecubital vein. The handgrip strength test will be performed to assess muscle strength. The SPPB test will be performed to determine physical condition. CT-muscle volumetry will be performed on existing preoperative CT scans to distinguish sarcopenic and non-sarcopenic patients and assignment to sarcopenic and non-sarcopenic groups. Diaphragm, quadriceps femoris and rectus abdominis ultrasound will be performed as previously described. Lung function testing will be performed at the bedside as described. A Health-related Quality of Life questionnaire will be filled out (HRQoL) (SF-36).

\section{- T2 (intraoperative sampling)}

Intraoperative tissue sampling of the m. rectus abdominis, samples will be snap frozen in liquid nitrogen in the operating theatre and stored at $-80^{\circ} \mathrm{C}$. Sample size will be approximately $1 \mathrm{~cm}^{3}$ and will be resected using non-electric scissors.

- T3 (day 1 postoperative)

Diaphragm, quadriceps femoris and rectus abdominis ultrasound will be performed. Handgrip strength test will be performed, and blood samples will be taken from the antecubital vein; serum or plasma will be processed and frozen.

- T4 (day 3 postoperative)

Diaphragm, quadriceps and rectus abdominis ultrasound will be performed. Handgrip strength test will be performed, and blood samples will be taken; serum or plasma will be processed and frozen.

- T5 (day 5 postoperative)

Diaphragm, quadriceps and rectus abdominis ultrasound will be performed. Handgrip strength test will be performed, and blood samples will be taken; serum or plasma will be processed and frozen.

- T6 (day 7 postoperative)

Diaphragm, quadriceps and rectus abdominis ultrasound will be performed. Handgrip strength test will be performed and blood samples will be taken; serum or plasma will be processed and frozen. HRQoL questionnaire will be filled out. The SPPB test will be performed.

- T7 (30 days after hospital discharge)

Diaphragm, quadriceps and rectus abdominis ultrasound will be performed. Handgrip strength test will be performed, and blood samples will be taken; serum or plasma will be processed and frozen. HRQoL questionnaire will be filled out. The SPPB test will be performed.

- T8 (6 months after surgery)

A telephone questionnaire will be performed containing a physical activity questionnaire, return to baseline work/activity questionnaire and a HRQoL questionnaire (SF36).

\section{Setting}

Recruitment of patients and subsequent sampling will be performed tertiary university hospital in Germany, Uniklinik Rheinish Westphälische Technische Hochschule (RWTH) Aachen.

\section{STATISTICAL ANALYSIS}

\section{Power analyses}

No data regarding speckle imaging variables (strain or strain rate) among different patient groups are available. However, Deniz et al recently investigated differences in lung function between older sarcopenic and non-sarcopenic patients by measuring diaphragm thickness. ${ }^{21}$ We calculated the expected effect size based on mean diaphragm thickness values and SD after forced expiration of the aforementioned study. Based on a mean FT in the non-sarcopenic group $(\mathrm{n}=30)$ of $1.5 \mathrm{~mm}(\mathrm{SD} 0.7)$ and $1.1 \mathrm{~mm}(\mathrm{SD} \mathrm{0.4)}$ in the sarcopenic group $(n=30)$, we calculated an effect size of 0.70 . Considering an alpha of 0.05 and a beta of 0.80 , a total sample size of 66 patients (33 patients per group) will be needed.

Analysis of primary and secondary outcome parameters SPSS will be used for statistical analysis (IBM, Released 2013, IBM SPSS Statistics for Macintosh, V.22.0.). A 


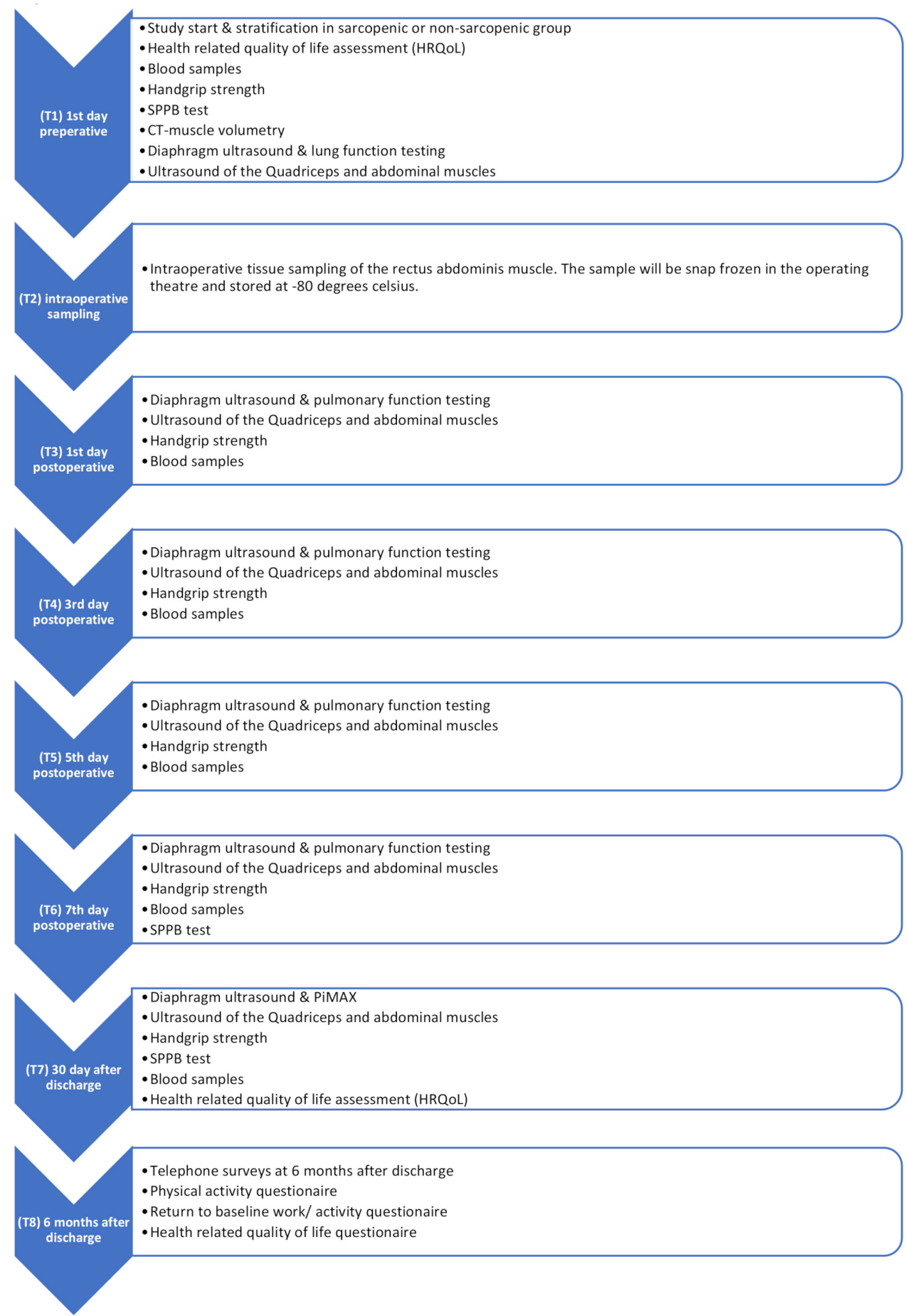

Figure 1 Flow chart showing study time plan from T1 1-day preoperative to T8 6 months after discharge. SPPB, short physical performance battery.

two-tailed $\mathrm{p}<0.05$ will be considered statistically significant. To allow comparisons between groups, data will be tested for normal distribution, and appropriate statistical tests will be applied, potentially including Student's t-test, Mann-Whitney U test, analysis of variance, Kruskal-Wallis test, $\chi^{2}$ test or Fisher's exact test. Normally distributed continuous variables will be evaluated as mean values and SD. Non-normally distributed continuous variables will be evaluated as median and range.

\section{PATIENT AND PUBLIC INVOLVEMENT}

No patients involved

\section{ETHICS AND DISSEMINATION}

The protocol was ethically approved by the official Independent Medical Ethical Committee of the Uniklinik (RWTH) Aachen (reference EK309-18) in July 2019. Written informed consent will be obtained from all participants. The study will be performed in accordance 
with the principles of the Declaration of Helsinki, as well as the guidelines of Good Clinical Practice. Recruitment started in the first quarter of 2020, and recruitment is currently ongoing. Patients deemed eligible for enrolment are initially recruited by their surgeon at the time of approval for surgery. If interested in participation, the patient will be contacted by the researcher and given detailed information about the study, in both oral and written form. After a 2-week period, the subjects are contacted to obtain informed consent, and then they will be officially enrolled in the study.

\section{DISCUSSION}

Sarcopenia is characterised by concurrent hypermetabolism, hypercatabolism and hypoanabolism which aggravate weight loss and are provoked by tumour induced systemic inflammation and catabolic factors party mediated by the central nervous system. ${ }^{39}$ Irrespective of the cause, sarcopenia has been shown to have a significant negative impact on short-term and long-term outcome following a range of oncological treatments. ${ }^{13740-45}$ Not only long-term outcome has been shown to be impacted by sarcopenia. Postoperative complications (morbidity) have profound impact on the burden of disease and suffering following surgical treatment. ${ }^{41} 42$ 46-48 Pulmonary morbidity, especially postoperative pneumonia, is of particular importance and has been shown to prolong hospital admission and increase in-hospital mortality following a range of surgical interventions, most notably after major abdominal and upper gastrointestinal surgery. ${ }^{11-15}$

The results of this study will contribute to the understanding of the role of the diaphragm in pulmonary morbidity following liver resection and will provide insight into the role of sarcopenia in pulmonary morbidity. To our knowledge, this approach has not previously been implemented for the investigation of the impact of abdominal surgery or sarcopenia and wasting disorders on diaphragm function.

We hypothesise that the results of this study will contribute to identifying patients at risk of pulmonary complications following liver resection. Identification of these at-risk patients is of great importance for the implementation of improved preconditioning of patients, thus potentially contributing to the reduction of postoperative pulmonary morbidity in the future.

'Is Sarcopenia a Risk Factor for Reduced Diaphragm Function Following Hepatic Resection, a Study Protocol for a Prospective Observational Study', is a study currently recruiting patients.

\section{Author affiliations}

${ }^{1}$ Department of General, Hepatobiliary and Transplant Surgery, Uniklinik RWTHAachen, Aachen, Germany

${ }^{2}$ Department of Intensive Care Medicine and Intermediate Care, Uniklinik RWTHAachen, Aachen, Germany

${ }^{3}$ Department of Surgery, Maastricht Universitair Medisch Centrum (MUMC+), Maastricht, Netherlands
${ }^{4}$ NUTRIM School of Nutrition and Translational Research In Metabolism, Maastricht University, Maastricht, Netherlands

${ }^{5}$ Department of Anaesthesiology, Uniklinik RWTH-Aachen, Aachen, Germany

Contributors GvdK: study concept, study design, writing protocol. SSR: study design, writing protocol. CS: study design, writing protocol. SJJF: expert support speckle imaging ultrasound. SW: imaging, physical activity measurements and ultrasound. UPN: study design. SWMOD: study concept, study design, writing protocol. CSB: study concept, study design, writing protocol. AL: data acquisition and study management.

Funding The authors have not declared a specific grant for this research from any funding agency in the public, commercial or not-for-profit sectors.

Competing interests None declared.

Patient consent for publication Not applicable.

Provenance and peer review Not commissioned; externally peer reviewed.

Supplemental material This content has been supplied by the author(s). It has not been vetted by BMJ Publishing Group Limited (BMJ) and may not have been peer-reviewed. Any opinions or recommendations discussed are solely those of the author(s) and are not endorsed by BMJ. BMJ disclaims all liability and responsibility arising from any reliance placed on the content. Where the content includes any translated material, BMJ does not warrant the accuracy and reliability of the translations (including but not limited to local regulations, clinical guidelines, terminology, drug names and drug dosages), and is not responsible for any error and/or omissions arising from translation and adaptation or otherwise.

Open access This is an open access article distributed in accordance with the Creative Commons Attribution Non Commercial (CC BY-NC 4.0) license, which permits others to distribute, remix, adapt, build upon this work non-commercially, and license their derivative works on different terms, provided the original work is properly cited, appropriate credit is given, any changes made indicated, and the use is non-commercial. See: http://creativecommons.org/licenses/by-nc/4.0/.

ORCID iDs

Gregory van der Kroft http://orcid.org/0000-0002-4847-4067

Sebastian Johannes Johannes Fritsch http://orcid.org/0000-0002-8350-8584

\section{REFERENCES}

1 Bachmann J, Heiligensetzer M, Krakowski-Roosen $\mathrm{H}$, et al. Cachexia worsens prognosis in patients with resectable pancreatic cancer. $J$ Gastrointest Surg 2008;12:1193-201.

2 van Dijk DPJ, Bakens MJAM, Coolsen MME, et al. Low skeletal muscle radiation attenuation and visceral adiposity are associated with overall survival and surgical site infections in patients with pancreatic cancer. J Cachexia Sarcopenia Muscle 2017;8:317-26.

3 van Vledder MG, Levolger S, Ayez N, et al. Body composition and outcome in patients undergoing resection of colorectal liver metastases. Br J Surg 2012;99:550-7.

4 Reisinger KW, van Vugt JLA, Tegels JJW, et al. Functional compromise reflected by sarcopenia, frailty, and nutritional depletion predicts adverse postoperative outcome after colorectal cancer surgery. Ann Surg 2015;261:345-52.

5 van der Kroft G, van Dijk DPJ, Rensen SS, et al. Low thoracic muscle radiation attenuation is associated with postoperative pneumonia following partial hepatectomy for colorectal metastasis. HPB 2020;22:1011-9.

6 Moon JH, Kong MH, Kim HJ. Implication of sarcopenia and sarcopenic obesity on lung function in healthy elderly: using Korean National health and nutrition examination survey. J Korean Med Sci 2015;30:1682-8.

7 Ida S, Watanabe M, Yoshida N, et al. Sarcopenia is a predictor of postoperative respiratory complications in patients with esophageal cancer. Ann Surg Oncol 2015;22:4432-7.

8 Maeda K, Akagi J. Muscle mass loss is a potential predictor of 90day mortality in older adults with aspiration pneumonia. J Am Geriatr Soc 2017;65:e18-22.

9 Makiura D, Ono R, Inoue J, et al. Preoperative sarcopenia is a predictor of postoperative pulmonary complications in esophageal cancer following esophagectomy: a retrospective cohort study. $J$ Geriatr Oncol 2016;7:430-6.

10 Nishigori T, Okabe H, Tanaka E, et al. Sarcopenia as a predictor of pulmonary complications after esophagectomy for thoracic esophageal cancer. J Surg Oncol 2016;113:678-84. 
11 Serpa Neto A, Hemmes SNT, Barbas CSV, et al. Incidence of mortality and morbidity related to postoperative lung injury in patients who have undergone abdominal or thoracic surgery: a systematic review and meta-analysis. Lancet Respir Med 2014;2:1007-15.

12 Haines KJ, Skinner EH, Berney S, et al. Association of postoperative pulmonary complications with delayed mobilisation following major abdominal surgery: an observational cohort study. Physiotherapy 2013;99:119-25

13 Fleisher LA, Linde-Zwirble WT. Incidence, outcome, and attributable resource use associated with pulmonary and cardiac complications after major small and large bowel procedures. Perioper Med 2014;3:7.

14 Fernandez-Bustamante A, Frendl G, Sprung J, et al. Postoperative pulmonary complications, early mortality, and hospital stay following Noncardiothoracic surgery: a multicenter study by the perioperative research network Investigators. JAMA Surg 2017;152:157-66.

15 Canet J, Gallart L, Gomar C, et al. Prediction of postoperative pulmonary complications in a population-based surgical cohort. Anesthesiology 2010;113:1338-50.

16 Shander A, Fleisher LA, Barie PS, et al. Clinical and economic burden of postoperative pulmonary complications: patient safety Summit on definition, risk-reducing interventions, and preventive strategies. Crit Care Med 2011:39:2163-72.

17 LAS VEGAS investigators. Epidemiology, practice of ventilation and outcome for patients at increased risk of postoperative pulmonary complications: LAS VEGAS - an observational study in 29 countries. Eur J Anaesthesiol 2017;34:492-507.

18 de Boer MT, Boonstra EA, Lisman T, et al. Role of fibrin sealants in liver surgery. Dig Surg 2012;29:54-61.

19 Fearon K, Strasser F, Anker SD, et al. Definition and classification of cancer cachexia: an international consensus. Lancet Oncol 2011;12:489-95.

20 Roberts BM, Ahn B, Smuder AJ, et al. Diaphragm and ventilatory dysfunction during cancer cachexia. Faseb J 2013;27:2600-10.

21 Deniz O, Coteli S, Karatoprak NB, et al. Diaphragmatic muscle thickness in older people with and without sarcopenia. Aging Clin Exp Res 2021;33:573-80.

22 Greising SM, Mantilla CB, Gorman BA, et al. Diaphragm muscle sarcopenia in aging mice. Exp Gerontol 2013;48:881-7.

23 Greising SM, Mantilla CB, Medina-Martínez JS, et al. Functional impact of diaphragm muscle sarcopenia in both male and female mice. Am J Physiol Lung Cell Mol Physiol 2015;309:L46-52.

24 Criswell DS, Shanely RA, Betters JJ, et al. Cumulative effects of aging and mechanical ventilation on in vitro diaphragm function. Chest 2003;124:2302-8.

25 Farghaly S, Hasan AA. Diaphragm ultrasound as a new method to predict extubation outcome in mechanically ventilated patients. Aust Crit Care 2017;30:37-43.

26 Goligher EC, Dres M, Fan E, et al. Mechanical Ventilation-induced diaphragm atrophy strongly impacts clinical outcomes. Am J Respir Crit Care Med 2018;197:204-13.

27 Matamis D, Soilemezi E, Tsagourias M, et al. Sonographic evaluation of the diaphragm in critically ill patients. technique and clinical applications. Intensive Care Med 2013;39:801-10.

28 Ayoub J, Cohendy R, Prioux J, et al. Diaphragm movement before and after cholecystectomy: a sonographic study. Anesth Analg 2001;92:755-61.

29 Lerolle N, Guérot E, Dimassi S, et al. Ultrasonographic diagnostic criterion for severe diaphragmatic dysfunction after cardiac surgery. Chest 2009;135:401-7.
30 Hellyer NJ, Andreas NM, Bernstetter AS, et al. Comparison of diaphragm thickness measurements among postures via ultrasound imaging. $P m$ R 2017;9:21-5.

31 DiNino E, Gartman EJ, Sethi JM, et al. Diaphragm ultrasound as a predictor of successful extubation from mechanical ventilation. Thorax 2014;69:431-5.

32 Goligher EC, Laghi F, Detsky ME, et al. Measuring diaphragm thickness with ultrasound in mechanically ventilated patients: feasibility, reproducibility and validity. Intensive Care Med 2015;41:642-9.

33 Oppersma E, Hatam N, Doorduin J, et al. Functional assessment of the diaphragm by speckle tracking ultrasound during inspiratory loading. J Appl Physiol 2017;123:1063-70.

34 Amundsen BH, Helle-Valle T, Edvardsen T, et al. Noninvasive myocardial strain measurement by speckle tracking echocardiography: validation against sonomicrometry and tagged magnetic resonance imaging. J Am Coll Cardiol 2006;47:789-93.

35 Bahat G, Tufan A, Tufan F, et al. Cut-Off points to identify sarcopenia according to European Working group on sarcopenia in older people (EWGSOP) definition. Clin Nutr 2016;35:1557-63.

36 Mourtzakis M, Prado CMM, Lieffers JR, et al. A practical and precise approach to quantification of body composition in cancer patients using computed tomography images acquired during routine care. Appl Physiol Nutr Metab 2008;33:997-1006.

37 Martin L, Birdsell L, Macdonald N, et al. Cancer cachexia in the age of obesity: skeletal muscle depletion is a powerful prognostic factor, independent of body mass index. $\mathrm{J}$ Clin Oncol 2013;31:1539-47.

38 Cruz-Jentoft AJ, Baeyens JP, Bauer JM, et al. Sarcopenia: European consensus on definition and diagnosis: report of the European Working group on sarcopenia in older people. Age Ageing 2010;39:412-23.

39 Fearon K, Arends J, Baracos V. Understanding the mechanisms and treatment options in cancer cachexia. Nat Rev Clin Oncol 2013;10:90-9.

40 Dewys WD, Begg C, Lavin PT, et al. Prognostic effect of weight loss prior tochemotherapy in cancer patients. Am J Med 1980;69:491-7.

41 Joglekar S, Asghar A, Mott SL, et al. Sarcopenia is an independent predictor of complications following pancreatectomy for adenocarcinoma. J Surg Oncol 2015;111:771-5.

42 Lieffers JR, Bathe OF, Fassbender K, et al. Sarcopenia is associated with postoperative infection and delayed recovery from colorectal cancer resection surgery. Br J Cancer 2012;107:931-6.

43 Norman K, Pichard C, Lochs H, et al. Prognostic impact of diseaserelated malnutrition. Clin Nutr 2008;27:5-15.

44 Tisdale MJ. Mechanisms of cancer cachexia. Physiol Rev 2009;89:381-410.

45 Uomo G, Gallucci F, Rabitti PG. Anorexia-Cachexia syndrome in pancreatic cancer: recent development in research and management. JOP 2006;7:157-62.

46 Gani F, Buettner S, Margonis GA, et al. Sarcopenia predicts costs among patients undergoing major abdominal operations. Surgery 2016;160:1162-71.

47 Kuritzkes BA, Pappou EP, Kiran RP, et al. Visceral fat area, not body mass index, predicts postoperative 30 -day morbidity in patients undergoing colon resection for cancer. Int $\mathrm{J}$ Colorectal Dis 2018;33:1019-28.

48 Takagi K, Yoshida R, Yagi T, et al. Radiographic sarcopenia predicts postoperative infectious complications in patients undergoing pancreaticoduodenectomy. BMC Surg 2017;17:64. 